\title{
MONITORAMENTO DA RESISTÊNCIA DE TETRANYCHUS URTICAE KOCH (ACARI: TETRANYCHIDAE) A ABAMECTIN E FENPYROXIMATE EM DIVERSAS CULTURAS NO ESTADO DE SÃO PAULO
}

\author{
M.E. Sato*, M.Z. da Silva**, R.B. da Silva, M.F. de Souza Filho, A. Raga*
}

Instituto Biológico, Centro Experimental Central, CP 70, CEP 13001-970, Campinas, SP, Brasil. E-mail: mesato @biologico.sp.gov.br

\section{RESUMO}

\begin{abstract}
A resistência de ácaro rajado, Tetranychus urticae Koch, a acaricidas tem sido registrada em diversos países, inclusive no Brasil. O estudo teve como objetivo detectar a resistência de T. urticae aos acaricidas abamectin e fenpyroximate, e avaliar a frequência de resistência a esses compostos em áreas comerciais de diversas culturas no Estado de São Paulo. Foram estabelecidas as concentrações discriminatórias de 4,79 mg de ingrediente ativo (i.a.)/L para abamectin e de 46,3 mg de i.a./ $\mathrm{L}$ para fenpyroxymate, para o monitoramento da resistência de $T$. urticae a esses acaricidas. $\mathrm{O}$ monitoramento foi realizado coletando-se 29 populações deácaros emáreas comerciais de diferentes culturas (morango, feijão, figo, framboesa, mamão, pêssego, tomate, crisântemo, áster, tango), em 15 municípios do Estado de São Paulo. Arenas confeccionadas com folha de feijão foram infestadas com ácaros T. urticae e pulverizadas com um dos acaricidas avaliados, na sua respectiva concentração discriminatória, em torre de Potter. As porcentagens de sobrevivência foram avaliadas 48 horas após a aplicação. Os resultados indicaram grande variabilidade entre as populações com relação à suscetibilidade a abamectin e fenpyroximate. Foram observadas populações com frequências de resistência entre zero e $81,3 \%$ para abamectin; e entre zero e $94,5 \%$ para fenpyroximate. As maiores frequências de resistência foram observadas em populações coletadas de ornamentais.
\end{abstract}

PALAVRAS-CHAVE: Ácaro rajado, resistência a acaricida, morango, crisântemo.

\section{ABSTRACT}

MONITORING OF RESISTANCETO ABAMECTIN ANDFENPYROXIMATEINTETRANYCHUS URTICAE KOCH (ACARI: TETRANYCHIDAE) IN SEVERAL CROPS IN THE STATE OF SÃO PAULO, BRAZIL. The acaricide resistance in Tetranychusurticae Koch has been registered in several countries including Brazil. The objective of this study was to detect the resistance of T. urticae to the acaricides abamectin and fenpyroximate, and to evaluate the resistancefrequency in commercial fields of several crops in the state of São Paulo. Discriminating concentrations of $4.79 \mathrm{mg}$ of active ingredient (A.I.) / L for abamectin and of $46.3 \mathrm{mg}$ of A.I./ L for fenpyroximate were established for monitoring T. urticae resistance to these acaricides. Twenty nine mite populations were collected from commercial fields of different crops (strawberry, bean, fig, raspberry, papaya, peach, tomato, chrysanthemum, aster, goldenrod), in 15 counties in the state of São Paulo. Bean-leaf-disc arenas were infested with $T$. urticae mites and submitted to acaricide spraying at the discriminating concentration of each chemical, using a Potter spray tower. The percentages of survival were registered 48 hours after treatment. The results showed significant differences among populations in their responses to abamectin and fenpyroximate. Populations with frequencies of resistance from zero to $81.3 \%$ were observed for abamectin, and from zero to $94.5 \%$ for fenpyroximate. The highest frequencies of resistance were registered from populations collected at ornamental plants.

KEY WORDS: Two-spotted spider mite, acaricide resistance, strawberry, chrysanthemum.

\section{INTRODUÇÃO}

O ácaro rajado, Tetranychus urticae Koch (Acari: Tetranychidae), considerado de grande importância econômica em todo o mundo, tem causado consideráveis prejuízos em diversas culturas no Brasil. Altas infestações podem causar descoloração de folhas, perda da capacidade fotossintética e, eventualmente,

\footnotetext{
*Bolsista do CNPq.

**Biólogo, bolsista de Doutorado da CAPES.
} 
a morte das folhas Chiavegato; Mischan, 1981; Flechtmann, 1985; Devine et al., 2001). O maior problema associado ao controle químico do ácaro rajado é seu elevado potencial reprodutivo e seu curto ciclo de vida, que favorecem o rápido desenvolvimento de resistência a acaricidas STUMPF; NAuen, 2001).

A resistência de T. urticae a acaricidas de diversos grupos químicos (ex.: dimetoato, dicofol, cyhexatin, hexythiazox, clofentezina, abamectin, fenpyroximate, chlorfenapyr) tem sido reportada em diversos países (CRANHAm; Helle, 1985; Herron etal., 1993; SA To et al., 1994; CAMPos et al., 1996; STUMPF \& Nauen, 2001; Stumpf; Nauen, 2002; Herron; Rophail, 2003). No Brasil, ainda são relativamente poucos os trabalhos sobre a resistência dessa praga a acaricidas (Chiavegato et al., 1983; SouZA Filho et al., 1994;SATO et al., 2000).

Abamectiné utilizado no Brasil para o controle de diversas espécies de insetos [ex.: Alabama argillacea (Hüebner), Phyllocnistis citrella Staiton, Tuta absoluta (Meyrick)] e ácaros [T. urticae, Polyphagotarsonemus latus (Banks), Phyllocoptrutaoleivora (Ashmead), Aceria guerreronis Keifer, Panonychus ulmi (Koch), Aculops lycopersici (Massee), Oligonychus ilicis (McGregor)], em várias culturas, tais como algodão, citros, coco, maçã, melancia, morango, pepino, tomate, café, batata e ornamentais. O acaricida fenpyroximate também é recomendado para o controle de ácaros [ex.: $T$. urticae, P. latus, A. gurreronis, P. ulmi, Brevipalpus phoenicis (Geijskes), P.oleivora], em diferentes culturas (ex.: morango, mamão, tomate, rosa, coco, maçã, café e citros) no país (AGROFIT, 2007).

Recentemente, alguns agricultores têm observado baixa eficiência de controle de T. urticae com a utilização de produtos como abamectin e fenpyroximate, em culturas como morangueiro e ornamentais, no Estado deSão Paulo. Uma das possíveis razões para o problema de controle é o desenvolvimento da resistência de ácaro rajado a acaricidas.

Oobjetivo do presente trabalho foi detectar a resistência e estimar a frequência de resistência de ácaros T. urticae a abamectin e fenpyroximate, em diversas culturas e localidades no Estado de São Paulo.

\section{MATERIAL E MÉTODOS}

O trabalho foi conduzido no Laboratório de Entomologia Econômica, do Centro Experimental Central do Instituto Biológico, em Campinas, SP.

Criação de ácaros: Os ácaros T. urticae foram criados sobre plantas de feijão-de-porco [Canavalia ensiformis (L.)] cultivadas em vasos no interior de bandejas plásticas, à temperatura de $25 \pm 1^{\circ} \mathrm{C}$, umidade relativa de $70 \pm 10 \%$ e fotofase de $14 \mathrm{~h}$.
Toxicidade de acaricidas: Os testes com os acaricidas abamectin (Vertimec ${ }^{\circledR} 18 \mathrm{CE}$ ) e fenpyroximate (Ortus ${ }^{\circledR}$ $50 \mathrm{SC}$ ) foram realizados baseando-se no método descrito por KNIGHT et al. (1990). Foram colocadas 20 fêmeas adultas de T. urticae em um disco de folha de feijão (C. ensiformis) de $4 \mathrm{~cm}$ de diâmetro, mantido sobre uma camada de algodão hidrófilo úmido em uma placa de Petri de $9 \mathrm{~cm}$ de diâmetro. A borda da folha foi coberta por uma estreita faixa de algodão úmido, formando uma barreira para evitar a fuga dos ácaros. Cada arena foi pulverizada em torre de Potter (Burkard Scientific, Rickmansworth, Herts, UK), calibrada a $68,9 \mathrm{kPa}$. Utilizou-se um volume de calda de $2 \mathrm{~mL}$, correspondendo a uma deposição média de resíduo úmido de $1,6 \mathrm{mg} / \mathrm{cm}^{2}$ da arena. Após o tratamento, os ácaros foram mantidos a $25 \pm$ $1^{\circ} \mathrm{C}, 70 \pm 10 \%$ de UR e fotofase de $14 \mathrm{~h}$. As avaliações do número de ácaros vivos e mortos foram conduzidas 48 h após o tratamento. Foram considerados mortos os ácaros que não conseguiam locomover-se por uma distância mínima equivalente ao comprimento do seu corpo, ao serem tocados com um pincel de pêlo macio.

Seleções artificiais com acaricidas: Foram realizadas seleções para resistência e suscetibilidade a abamectin e fenpyroxymate utilizando populações de T. urticae coletadas em cultivo comercial de morangueiro, em Atibaia, SP. As seleções para resistência e suscetibilidade a fenpyroximate (SATO et al., 2004) e abamectin (SATO et al., 2005) foram realizadas em condições de laboratório.

Nas seleções para resistência, fêmeas adultas de $T$. urticae foram colocadas em arenas de folha de feijão, realizando-se, em seguida, a pulverização com $2 \mathrm{~mL}$ de um dos acaricidas, em torre de Potter. Foram utilizadas concentrações crescentes de acaricida a cada ciclo de seleção, de forma que aproximadamente 30 a $40 \%$ dos ácaros sobrevivessem para formar as novas gerações.

As seleções para suscetibilidade a abamectin e fenpyroximate foram realizadas com as mesmas populações utilizadas para resistência, para cada produto. A finalidade dessas seleções foi remover das populações os genes responsáveis pela resistência aos acaricidas, e assim obter linhagens mais suscetíveis a estes produtos. Nas seleções para suscetibilidade, fêmeas acasaladas foram colocadas individualmente em arenas de folha de feijão e deixadas para ovipositar, por um período de $48 \mathrm{~h}$. Cada fêmea foi transferida para uma nova arena de folha de feijão e tratada com acaricida (torre dePotter). Concentrações decrescentes de acaricidas foram utilizadas a cada ciclo de seleção, causando mortalidades entre 30 a $40 \%$. Somente os descendentes correspondentes às fêmeas que morreram foram utilizados para formar as gerações posteriores. 
Foram realizadas cinco seleções para resistência e três para suscetibilidade a fenpyroximate, e cinco seleções para resistência e suscetibilidade ao abamectin.

Durante o processo de seleção com fenpyroximate, a razão de resistência $\left(\mathrm{CL}_{50} \mathrm{R}\right.$ dividido pela $\left.\mathrm{CL}_{50} \mathrm{~S}\right)$ passou de 1 (antes da seleção) para 2.910 vezes. A concentração letal $50 \%\left(\mathrm{CL}_{50}\right)$ de fenpyroximate passou de $111 \mathrm{mg}$ de i.a. (ingrediente ativo)/L (antes da seleção) para $10.900 \mathrm{mg}$ de i.a./L após a seleção para resistência. $\mathrm{Na}$ seleção para suscetibilidade, a $\mathrm{CL}_{50}$ passou de $111 \mathrm{mg}$ de i.a./L para 3,74 $\mathrm{mg}$ de i.a./L (SATO et al., 2004).

Com as seleções utilizando abamectin, a razão de resistência $\left(\mathrm{CL}_{50} \mathrm{R} / \mathrm{CL}_{50} \mathrm{~S}\right)$ passou de 1 para 342 vezes. $\mathrm{A} \mathrm{CL}_{50}$ de abamectin passou de $4,36 \mathrm{mg}$ dei.a./ L para 58,1 mg de i.a./L, após a seleção para resistência; e de 4,36 mg dei.a./L para 0,17 mg dei.a./L, após a seleção para suscetibilidade (SATO et al., 2005).

Concentrações discriminatórias de acaricidas: As concentrações discriminatórias de cada produto foram estimadas por meio da $\mathrm{CL}_{99}$ de cada produto, para a respectiva população suscetível selecionada. Concentrações discriminatórias seriam aquelas capazes de matar praticamente a totalidade dos ácaros suscetíveis, sem causar mortalidade nos ácaros resistentes. Essas concentrações discriminatórias serviram para o monitoramento da frequência de resistência em diversas populações de ácaros T. urticae.

A partir do estabelecimento da concentração discriminatória, foi possível monitorar a resistência a abamectin e fenpyroximate, baseando-se apenas na sobrevivência dos ácaros para uma única concentração de cada acaricida. De acordo com HallidAy \& BuRnHAM (1990), esse método é mais eficiente que o monitoramento da resistência baseado na estimativa da $\mathrm{CL}_{50}$, permitindo a detecção da resistência em populações em que a freqüência de indivíduos resistentes ainda se mostra baixa.

Monitoramento da resistência: foram coletadas populações de T. urticae em culturas comerciais de mamão (Carica papaya L.), morango (Fragaria sp.), feijão (Phaseolus vulgaris L.), figo (Ficus carica L.), framboesa (Rubus idaeus L.), pêssego (Prunus persica L.), tomate (Solanum lycopersicum L.), crisântemo (Chrysantemum sp.), áster (Aster sp.) e tango (Solidago sp.), nos municípios paulistas de Araras, Atibaia, Campinas, Campos do Jordão, Holambra, Jarinu, Jundiaí, Leme, Louveira, Monte Alegre do Sul, Pereiras, Piedade, Regente Feijó, Serra Negra e Socorro, totalizando 29 populações.

Após a coleta, os ácaros foram transferidos para plantas de feijão-de-porco, e mantidos por um período de 14 a 26 dias, em condições de laboratório (25 \pm $1^{\circ} \mathrm{C}, 70 \pm 5 \%$ de UR e fotofase de $14 \mathrm{~h}$ ). Os testes toxicológicos foram realizados seguindo-se a metodologia descrita anteriormente (pulverização direta sobre os ácaros). Os acaricidas foram pulverizados nas suas respectivas concentrações discriminatórias (abamectin a 4,79 $\mathrm{mg}$ de i.a./L e fenpyroxymate a $46,3 \mathrm{mg}$ de i.a./L).

Foram utilizados de 200 a 320 ácaros (4 repetições de 50 a 80 ácaros/arena), para a estimativa da freqüência de resistência de T. urticae, para cada população avaliada. As porcentagens de mortalidade para cada população de ácaro rajado foram corrigidas através da fórmula de Аввотт (1925), transformados para arc-sen $\sqrt{\mathrm{x} / 100}$. As médias de sobrevivência foram comparadas pelo teste de Tukey. O nível de significância dos testes foi de $\alpha=0,05$.

\section{RESULTADOS E DISCUSSÃO}

A avaliação de 29 populações de T. urticae de diversas culturas no Estado deSão Paulo indicou que a suscetibilidade das populações de ácaro rajado a abamectin e fenpyroximate mostrou-se bastante variável, com frequências de resistência entre zero e $94,5 \%$ (Tabela 1).

As populações coletadas de feijão, framboesa, pêssego e tomate foram suscetíveis a ambos os acaricidas. Foram registradas baixas porcentagens de sobrevivência $(\leq 5,0 \%)$ deácaros após o tratamento com um desses produtos, não diferindo estatisticamente $(p>0,05)$ da testemunha, representada pela linhagem suscetível (S), selecionada para suscetibilidade.

Para abamectin, 51,7\% das populações apresentaram uma porcentagem de sobrevivência igual ou inferior a 5,0\%, não diferindo estatisticamente $(p>$ $0,05)$ da linhagem suscetível de referência.

No caso de morangueiro, $73,3 \%$ das populações apresentaram frequências de resistência a abamectin abaixo de $10 \%$. Essa porcentagem relativamente alta de populações suscetíveis a abamectin, em morangueiro, pode estar associada à baixa estabilidade da resistência de T. urticae a abamectin. Estudos realizados em laboratório mostraram um restabelecimento da suscetibilidade da resistência em um período de seis meses, em populações com até $75 \%$ de ácaros resistentes, na ausência de pressão de seleção (SATOet al., 2005). No caso das áreas com cultivo de morango, no Estado de São Paulo, existe normalmente um período de vários meses sem aplicação de acaricidas, entre o final da colheita (com a eliminação das plantas de morango) e o início do aparecimento dos ácarospraga no cultivo do ano seguinte. Esse período, que pode chegar a seis ou sete meses, sem pressão de seleção, poderia ser suficiente para orestabelecimento da suscetibilidade a abamectin nas populações de $T$. urticae, nessa cultura. 
Tabela 1- Sobrevivência média (\%) ( \pm erro-padrão da média) de ácaros Tetranychus urticae de diferentes populações tratados com abamectin (a 4,79 mg de i.a./L) e fenpyroxymate (a 46,3 mg de i.a./L), nas suas respectivas concentrações discriminatórias.

\begin{tabular}{|c|c|c|c|c|c|}
\hline \multicolumn{2}{|c|}{ População } & \multicolumn{2}{|r|}{ Abamectin } & \multicolumn{2}{|c|}{ Fenpyroximate } \\
\hline Cultura & Local & $n^{*}$ & $\%$ Sobreviventes & $n$ & $\%$ Sobreviventes \\
\hline S (Testemunha) & - & 320 & $0,00 \pm 0,00 a^{* *}$ & 320 & $0,00 \pm 0,00 \mathrm{a}$ \\
\hline Morango & Atibaia I & 240 & $47,9 \pm 2,48 \mathrm{gh}$ & 240 & $22,9 \pm 3,43$ de \\
\hline Morango & Atibaia II & 240 & $2,50 \pm 1,08 a b$ & 240 & $14,2 \pm 2,60 \mathrm{~cd}$ \\
\hline Morango & Atibaia III & 240 & $4,17 \pm 1,08 \mathrm{bc}$ & 240 & $2,50 \pm 1,44 a b$ \\
\hline Morango & Louveira I & 240 & $0,42 \pm 0,42 a b$ & 240 & $0,83 \pm 0,48 \mathrm{a}$ \\
\hline Morango & Louveira II & 320 & $2,19 \pm 1,48 a b$ & 320 & $2,81 \pm 1,07 \mathrm{ab}$ \\
\hline Morango & Jarinu I & 200 & $0,00 \pm 0,00 \mathrm{a}$ & 200 & $6,68 \pm 2,64 a b c$ \\
\hline Morango & Jarinu II & 240 & $16,3 \pm 2,48$ de & 240 & $41,2 \pm 6,14$ ef \\
\hline Morango & Jundiaí & 240 & $0,42 \pm 0,42 a b$ & 240 & $2,92 \pm 1,42 \mathrm{ab}$ \\
\hline Morango & Monte Alegre do Sul I & 240 & $0,00 \pm 0,00 \mathrm{a}$ & 240 & $4,58 \pm 1,42 \mathrm{abc}$ \\
\hline Morango & Monte Alegre do Sul II & 320 & $0,62 \pm 0,62 a b$ & 320 & $0,31 \pm 0,31 \mathrm{a}$ \\
\hline Morango & Piedade I & 240 & $0,00 \pm 0,00 \mathrm{a}$ & 240 & $4,17 \pm 1,59 \mathrm{abc}$ \\
\hline Morango & Piedade II & 240 & $0,00 \pm 0,00 \mathrm{a}$ & 240 & $9,98 \pm 2,03 \mathrm{bcd}$ \\
\hline Morango & Serra Negra & 240 & $6,66 \pm 0,68 b c$ & 240 & $0,00 \pm 0,00 \mathrm{a}$ \\
\hline Morango & Socorro I & 240 & $19,2 \pm 1,98$ de & 240 & $3,75 \pm 0,79 a b c$ \\
\hline Morango & Socorro II & 240 & $68,7 \pm 3,99 \mathrm{hi}$ & 240 & $4,17 \pm 0,48 \mathrm{abc}$ \\
\hline Feijão & Monte Alegre do Sul & 240 & $0,62 \pm 0,62 \mathrm{ab}$ & 240 & $0,00 \pm 0,00 \mathrm{a}$ \\
\hline Figo & Campinas & 240 & $31,7 \pm 2,98$ efg & 240 & $42,5 \pm 2,59$ ef \\
\hline Framboesa & Campos do Jordão & 240 & $5,00 \pm 1,80 \mathrm{abc}$ & 240 & $2,08 \pm 0,80 a b$ \\
\hline Mamão & Regente Feijó & 240 & $13,2 \pm 3,23 \mathrm{~cd}$ & 240 & $0,00 \pm 0,00 \mathrm{a}$ \\
\hline Pêssego & Campinas & 200 & $0,83 \pm 0,83 a b$ & 200 & $4,00 \pm 1,41 \mathrm{abc}$ \\
\hline Tomate & Pereiras & 240 & $0,00 \pm 0,00 \mathrm{a}$ & 240 & $0,00 \pm 0,00 \mathrm{a}$ \\
\hline Crisântemo & Campinas & 240 & $41,7 \pm 1,79 \mathrm{fgh}$ & 240 & $51,7 \pm 5,58 \mathrm{f}$ \\
\hline Crisântemo & Araras & 240 & $50,4 \pm 3,14 \mathrm{~h}$ & 240 & $94,5 \pm 0,46 \mathrm{i}$ \\
\hline Crisântemo & Holambra I & 240 & $57,9 \pm 3,63 \mathrm{~h}$ & 240 & $75,8 \pm 5,11 \mathrm{~g}$ \\
\hline Crisântemo & Holambra II & 240 & $81,3 \pm 1,84 \mathrm{i}$ & 240 & $93,3 \pm 2,14 \mathrm{hi}$ \\
\hline Crisântemo & Holambra III & 320 & $3,33 \pm 1,80 \mathrm{ab}$ & 320 & $40,3 \pm 2,11$ ef \\
\hline Crisântemo & Leme & 240 & $30,0 \pm 4,41$ def & 240 & $80,4 \pm 3,29 \mathrm{gh}$ \\
\hline Áster & Holambra & 240 & $65,8 \pm 1,75 \mathrm{hi}$ & 240 & $91,4 \pm 1,52 \mathrm{hi}$ \\
\hline Tango & Holambra & 240 & $13,8 \pm 3,43 \mathrm{~cd}$ & 240 & $87,9 \pm 1,58$ ghi \\
\hline
\end{tabular}

${ }^{*} n=$ Número total de ácaros avaliados de cada população para cada produto.

** Médias originais seguidas da mesma letra, na coluna, não diferem significativamente entre si, a 5\%, pelo teste de Tukey.

Durante o período sem plantas de morango no campo, os ácaros rajados podem permanecer em diversas plantas hospedeiras, como plantas daninhas, vegetação de mata nativa e culturas comerciais ao redor. Apenas no caso da migração dos ácaros para culturas comerciais (ex.: feijão e tomate), pode haver alguma pressão de seleção com acaricidas, dificultando o restabelecimento da suscetibilidade. No ano seguinte, os ácaros rajados que permaneceram em plantas daninhas, ou outros hospedeiros que não recebem aplicação de acaricidas, podem colonizar as plantas de morango, formando uma população inicial suscetível a abamectin.

Apenas duas entre 15 populações coletadas de morangueiro apresentaram frequências de resistên- cia a abamectin iguais ou acima de $30 \%$, sendo uma de Atibaia (I) e a outra de Socorro (II). No caso de morangueiro deSocorro II, com $68,7 \%$ deácaros resistentes, os agricultores realizaram pulverizações a cada duas semanas, após o início das infestações de T. urticae. No momento da coleta dos ácaros, no final de setembro de 2006, já haviam sido realizadas nove aplicações de abamectin desde o plantio das mudas de morango.

No caso das ornamentais (crisântemo, áster e tango), o problema da resistência de $T$. urticae a abamectin mostra-se mais sério, com $75 \%$ das populações com frequências de resistência iguais ou acima de $30 \%$. Em uma das populações coletadas, de crisântemo (Holambra II), a porcentagem de ácaros resisten- 
tes foi de $81,3 \%$. Esses altos índices de resistência de ácaro rajado em ornamentais devem-seà alta frequência de pulverizações com acaricidas realizadas nessas culturas.

A resistência de T. urticaea abamectinjá havia sido registrada por diversos autores no exterior (CAMPOs et al., 1996; BEERs et al., 1998). STUMPF; NAUEN (2002), estudando as enzimas envolvidas na resistência de $T$. urticae a abamectin, observaram que as linhagens resistentes (NL-00 e COL-00) apresentavam as atividades de MFO (monooxigenases dependentes do citocromo P450) várias vezes mais altas que as da linhagem suscetívelGSS. O efeito tóxico de abamectin na linhagem resistente NL-00 foi fortemente aumentado pelos sinergistas PBO (butóxido de piperonila) e DEM (Dietil Maleato), sugerindo que MFO e GST (Glutationa S-transferases) podem estar envolvidas na resistência a abamectin (STUMPF; NAUEN, 2002).

Em relação ao fenpyroximate, $55,2 \%$ das populações apresentaram porcentagens de sobrevivência iguais ou abaixo de 4,58\%, não diferindo estatisticamente da população $S$, selecionada para suscetibilidade (Tabela 1).

Para morangueiro, apenas uma população, coletada em Jarinu, apresentou frequência de resistência acima de 30\%; duas outras populações (de Atibaia) apresentaram sobrevivências iguais ou acima de $14,2 \%$. As demais populações não diferiram estatisticamente da população $S$ de referência.

Ao contrário de abamectin, que tem sido o principal acaricida utilizado nas áreas de morangueiro avaliadas, fenpyroximate não tem sido utilizado com frequência nos últimos anos, nessa cultura.

O problema da resistência de T. urticae a fenpyroximate também foi mais sério em ornamentais, em relação às outras culturas, com todas as populações avaliadas apresentando frequências de resistência iguais ou acima de $40 \%$. Foram registradas porcentagens acima de $80 \%$ de ácaros resistentes em cinco das oito populações coletadas de ornamentais.

Essa alta frequência de resistência de ácaro rajado a fenpyroximate, em ornamentais, está provavelmente associada às características de herança da resistência de T. urticae ao acaricida. Estudos em laboratório indicaram que a resistência de ácaro rajado era monogênica eincompletamente dominante (com grau de dominância entre 0,511 e 0,580), características estas que são favoráveis à evolução da resistência (SATo et al., 2004).

Além disso, a resistência desse ácaro a fenpyroximate mostrou-se moderadamente estável, sendo necessários longos intervalos de tempo, sem pressão de seleção, para haver o restabelecimento da suscetibilidade. Em uma população com frequência inicial de resistência de $50 \%$, a porcentagem deácaros resistentes decresceu para 31,7\%, em um período de
12 meses sem aplicação de acaricidas (SATO et al., 2004).

Nas propriedades avaliadas no presente estudo, com exceção da área de crisântemo de Holambra III, as aplicações com acaricidas, em ornamentais, chegavam a ser realizadas em intervalos inferiores a uma semana, nos períodos de maior infestação de T. urticae. Na área Holambra III, o cultivo de crisântemo era conduzido sem o uso de acaricidas ou inseticidas, sendo que os ácaros-praga eram controlados por inimigos naturais de ocorrência natural [ex.: Phytoseiulus macropilis (Banks)] ou liberados. A porcentagem relativamente elevada $(40,3 \%)$ de ácaros resistentes a fenpyroximate, nessa área de crisântemo, provavelmente se deve à migração de ácaros resistentes a partir dos canteiros tratados com acaricidas, localizados ao redor dessa área.

Apesar de terem sido realizadas rotações de acaricidas de diferentes princípios ativos em ornamentais, em propriedades como de Araras e Holambra (I e II), devido à elevada frequência de aplicações de acaricidas, pulverizações repetidas de abamectin ou fenpyroximate acabavam sendo realizadas em intervalos abaixo do período necessário para o restabelecimento da suscetibilidade, com consequente aumento progressivo da frequência de resistência de T. urticae a esses acaricidas, nessas propriedades.

A utilização de acaricidas com resistência cruzada aos acaricidas estudados também pode ter influenciado no processo de evolução de resistência no campo. Existem registros de produtos, como pyridaben e dimetoato, que podem apresentar resistência cruzada a fenpyroximate (STUMPF; NAUEN, 2001; SATO et al., 2004). No caso de pyridaben (Sanmite ${ }^{\circledR}$ ), este acaricida apresenta o mesmo modo de ação que fenpyroximate, atuando como inibidor do complexo I da cadeia de transporte de elétrons (Hirata et al., 1995; WoOd et al., 1996).

Com relação à resistência de T.urticae a abamectin, estudos em laboratório indicaram possível resistência cruzada entre abamectin e milbemectin (SAToetal., 2005). Esses dois compostos apresentam mecanismos de ação semelhantes. Ambos são agonistas de GABA (ácido gama amino butírico) e afetam a abertura dos canais de cloro, levando à paralisia e morte dos ácaros (SHoop et al., 1995; BloOMQuist, 2001). Assim sendo, a utilização desses produtos em sistema de rotação pode favorecer o aumento da frequência de resistência aos dois acaricidas. Esse problema pode vir a ser relevante em culturas como crisântemo e rosa, para as quais, milbemectineabamectin sãoregistrados (AGROFIT , 2008) e têm sido utilizados com frequência.

Ainda há necessidade de estudos mais detalhados sobre mecanismos de resistência para confirmar a resistência cruzada entre milbemectin e abamectin em T. urticae. 
Embora algumas populações como de áster (de Holambra) ecrisântemo (de Holambra II) apresentem altas frequências de resistência tanto para fenpyroximate como para abamectin, não há indícios de resistência cruzada entre os dois compostos (SATO et al., 2004; SАTO et al., 2005). Esse fato é confirmado neste estudo, pois algumas populações apresentaram alta frequência de resistência a um acaricida e baixa frequência ao outro acaricida. Um exemplo é a população de tango, que apresentou apenas $13,8 \%$ de ácaros resistentes a abamectin; no entanto, a porcentagem de ácaros resistentes a fenpyroximate foi de $87,9 \%$. Um exemplo diferente é a população coletada de morangueiro na área de Socorro II, com $68,7 \%$ de ácaros resistentes a abamectin e apenas $4,17 \%$ de ácaros resistentes a fenpyroximate.

O presente estudo mostrou que já existem diversas populações de ácaro rajado resistentes a acaricidas, como abamectin e fenpyroximate, em culturas como morangueiro e ornamentais, no Estado de São Paulo, ondea pressão de seleçãocomacaricidaséintensa. Para essas culturas, a utilização de métodos alternativos ao controle químico deve ser incentivada para minimizar o problema da resistência a acaricidas. Nesse contexto, autilizaçãodeácaros predadoresdafamíliaPhytoseiidae pareceserumaalternativa promissora (Mc MURTRY,C ROFT, 1997; SAто et al., 2007). Esses predadores, quando em altas populações, podem propiciar a redução populacional desses ácaros-praga, reduzindo a necessidadedeaplicações deacaricidase, consequentemente, reduzindo a pressão de seleção sobre esses organismos.

\section{AGRADECIMENTOS}

Os autores agradecem à FAPESP (Fundação de Amparo à Pesquisa do Estado de São Paulo), pelo suporte financeiro desta pesquisa (Processos: 2005/ 04454-0 e 2007/08612-4). Ao IRAC-BR (Comitê Brasileiro de Ação a Resistência a Inseticidas), pelos recursos necessários para o pagamento de bolsa de iniciação científica a Rodrigo Buratto da Silva. Ao CNPq (Conselho Nacional de Desenvolvimento Científico e Tecnológico) pelas bolsas de Produtividade em Pesquisa ao primeiro e último autor.

\section{REFERÊNCIAS}

ABBOTT, W.S. A method for computing the effectiveness of an insecticide. Journal of Economic Entomology, v.18, p.265-267, 1925.

AGROFIT - Sistema de Agrotóxicos Fitossanitários. (Brasil). Registro de agrotóxicos e afins Disponível em: <http://agrofit.agricultura.gov.br/agrofit_cons/ principal_agrofit_cons>. Acesso em: 20 fev. 2008.
BEERS, E.H.; RIEDL, H.; DUNLEY, J.E. Resistance to abamectin and reversion to susceptibility to fenbutatin oxide in spider mite (Acari: Tetranychidae) populations in the Pacific Northwest. Journal of Economic Entomology, v.91, p.352-360, 1998.

BLOOMQUIST, J.R. GABA and glutamate receptors as biochemical sites for insecticide action, In: ISHAAYA, I. (Ed.). Biochemical sites of insecticide action and resistance. New York: Springer, 2001. p.17-41.

CAMPOS, F.; KRUPA, D.A.; DYBAS, R.A. Susceptibility of populations of twospotted spider mites (Acari: Tetranychidae) from Florida, Holland, and the Canary Islands to abamectin and characterization of abamectin resistance. Journal of Economic Entomology, v.89, p.594601, 1996.

CHIAVEGATO, L.G.; MISCHAN, M.M. Efeito do ácaro Tetraychus (T.) urticae (Koch, 1836), 1963 (Acari: Tetranychidae) na produção no morangueiro (Fragaria spp.) cv. 'Campinas'. Científica, v.9, n.2, p.257-266, 1981.

CHIAVEGATO, L.G.; MISCHAN, M.M.; COTAS, M.P. Resistência do ácaro-rajado Tetranychus (T.) urticae (Koch, 1836) (Acari: Tetranychidae) proveniente de diferentes regiões algodoeiras aos acaricidas. Científica, v.11, p.57-62, 1983.

CRANHAM, J.E.; HELLE, W. Pesticide resistance in Tetranychidae. In: HELLE, W.; SABELIS, M.W. (Ed.). Spider mites: their biology, natural enemies, and control. Amsterdam: Elsevier, 1985. v.1B, p.405-421.

DEVINE, G.J.; BARBER, M.; DENHOLM, I. Incidence and inheritance of resistance to METI-acaricides in European strains of the two-spotted spider mite (Tetranychus urticae) (Acari: Tetranychidae). Pest Management Science, v.57, p.443-448, 2001.

FLECHTMANN, C.H.W. Ácaros de importância agrícola. São Paulo: Livraria Nobel, 1985. 189p.

HALLIDAY, W.R.; BURNHAM, K.P. Choosing the optimal diagnostic dose for monitoring insecticide resistance. Journal of Economic Entomology, v.83, p.1.151-1.159, 1990.

HERRON, G.A.; EDGE, V.; ROPHAIL, J. Clofentezine and hexythiazox resistance in Tetranychus urticae Koch in Australia. Experimental and Applied Acarology, v.17, p.433-440, 1993.

HERRON, G.A.; ROPHAIL, J. First detection of chlorfenapyr (Secure ${ }^{\circledR}$ ) resistance in two-spotted spider mite (Acari: Tetranychidae) from nectarines in an Australia orchard. Experimental and Applied Acarology, v.31, n.1-2, p.131-134, 2003.

HIRATA, K.; KAWAMURA, T.; KUDO, M.; IGARASHI, $H$. Development of a new acaricide, pyridaben. Journal of Pesticide Science, v.20, p.177-179, 1995. 
KNIGHT, A. L.; BEERS, E.H.; HOYT, S.C.; RIEDL, H. Acaricide bioassay with spider mites (Acari: Tetranychidae) on pome fruits: evaluation of methods and selection of discrimination concentrations for resistance monitoring. Journal of Economic Entomology, v.83, p.1.752-1.760, 1990 .

McMURTRY, J.A.; CROFT, B.A. Life styles of phytoseiid mites and their roles as biological control agents. Annual Review of Entomology, v.42, p.291-321, 1997.

SATO, M.E.; SUPLICY FILHO, N.; SOUZA FILHO, M.F. DE; TAKEMATSU, A.P. Resistência do ácaro rajado Tetranychus urticae (Koch, 1836) (Acari: Tetranychidae) a diversos acaricidas em morangueiro (Fragaria sp.) nos municípios de Atibaia-SP e Piedade-SP. Ecossistema, v.19, p.40-46, 1994.

SATO, M.E.; PASSEROTTI, C.M.; TAKEMATSU, A.P.; SOUZA FILHO, M.F. de; POTENZA, M.R.; SIVIERI, A.P. Resistência de Tetranychus urticae (Koch, 1836) a acaricidas, em pessegueiro (Prunus persica (L.) Batsch) em Paranapanema e Jundiaí-SP. Arquivos do Instituto Biológico, São Paulo, v.67, n.1, p.117-123, 2000.

SATO, M.E.; MIYATA, T.; SILVA, M. da; RAGA, A.; SOUZA FILHO, M.F. de Selections for fenpyroximate resistance and susceptibility, and inheritance, crossresistance and stability of fenpyroximate resistance in Tetranychus urticae Koch (Acari: Tetranychidae). Applied Entomology and Zoology, v.39, p.293-302, 2004.

SATO, M.E.; SILVA, M.Z. da; RAGA, A.; SOUZA FILHO, M.F. de Abamectin resistance in Tetranychus urticae Koch (Acari: Tetranychidae): selection, cross-resistance and stability of resistance. Neotropical Entomology, v.34, n.6, p.1-8, 2005.
SATO, M.E.; SILVA, M.Z. DA; SOUZA FILHO, M.F. DE; MATIOLI, A.L.; RAGA, A. Management of Tetranychus urticae (Acari: Tetranychidae) in strawberry fields with Neoseiulus californicus (Acari: Phytoseiidae) and acaricides. Experimental and Applied Acarology, v.42, p.107-120, 2007.

SHOOP, W.L.; MROZIK, H.; FISHER, M. Structure and activity of avermectins and milbemycins in animal health. Veterinary Parasitology, v.59, p.139-156, 1995.

SOUZA FILHO, M.F. de; SUPLICY FILHO, N.; SATO, M.E.; TAKEMATSU, A.P. Suscetibilidade do ácarorajado proveniente de Pilar do Sul-SP, a diversos acaricidas. Pesquisa Agropecuária Brasileira, v.29, p.1.1871.192, 1994.

STUMPF, N.; NAUEN, R. Cross-resistance, inheritance, and biochemistry of mitochondrial electron transport inhibitor-acaricide resistance in Tetranychus urticae (Acari: Tetranychidae). Journal of Economic Entomology, v.94, p.1.577-1.583, 2001.

STUMPF, N.; NAUEN, R. Biochemical markers linked to abamectin resistance in Tetranychus urticae (AcariTetranychidae). Pesticide Biochemistry and Physiology, v.72, p.111-121, 2002.

WOOD, E.; LATLI, B.; CASIDA, J.E. Fenazaquin acaricide specific binding sites in NADH: ubiquinone oxidoreductase and apparently the ATP synthase stalk. Pesticide Biochemistry and Physiology, v.54, p.135-145, 1996.

Recebido em $14 / 2 / 08$

Aceito em 8/5/09 